\title{
INTEGRAL: ICT-platform based Distributed Control in electricity grids with a large share of Distributed Energy Resources and Renewable Energy Sources
}

\author{
Gerard Peppink ${ }^{1}$, René Kamphuis ${ }^{1}$, Koen $\mathrm{Kok}^{1}$, Aris Dimeas ${ }^{2}$, Evangelos \\ Karfopoulos $^{2}$, Nikos Hatziargyriou ${ }^{2}$, Nourédine Hadjsaid ${ }^{3}$, Raphael Caire ${ }^{3}$, Rune \\ Gustavsson $^{4}$, Josep M. Salas ${ }^{5}$, Hugo Niesing ${ }^{5}$, Jörgen van der Velde ${ }^{6}$, Llani Tena ${ }^{7}$, \\ Frits Bliek $^{8}$, Marcel Eijgelaar ${ }^{9}$, Luc Hamilton ${ }^{10}$, Hans Akkermans ${ }^{10}$ \\ ${ }_{1}$ Energy Research Center of the Netherlands ECN, The Netherlands \\ 2 ICCS, NTUA, Greece 3 INPG Grenoble / IDEA, France \\ ${ }_{4}$ Blekinge Institute of Technology BTH, Sweden ${ }_{5}$ WattPic, Spain \\ ${ }_{6}$ Humiq, The Netherlands ${ }_{7}$ CRIC, Spain ${ }_{8}$ KEMA, The Netherlands \\ 9 Essent New Energy, The Netherlands, 10 EnerSearch, Sweden \\ Contact: peppink@ecn.nl
}

\begin{abstract}
The European project INTEGRAL aims to build and demonstrate an industry-quality reference solution for DER aggregation-level control and coordination, based on commonly available ICT components, standards, and platforms. To achieve this, the Integrated ICT-platform based Distributed Control (IIDC) is introduced. The project includes also three field test site installations in the Netherlands, Spain and France, covering normal, critical and emergency grid conditions.
\end{abstract}

Keywords: Smart Grids, DER/RES integration, Distributed Control, ICT, multi-agent systems

\section{Introduction}

With large shares of Distributed Energy Resources (DER) and Renewable Energy Sources (RES) that are expected in coming decades as a consequence of reaching EU policy targets, important challenges will have to be met with regard to controllability and optimal integration in Electric Power Systems of the EU and its Member States.

The EU project INTEGRAL (http://www.integral-eu.com) aims to build and demonstrate an industry-quality reference solution for DER aggregation-level control and coordination, based on commonly available ICT components, standards, and platforms.

To achieve this Integrated ICT-platform based Distributed Control (IIDC) solution (see Sec. 2), the INTEGRAL project is taking the following steps:

- Define Integrated Distributed Control as a unified and overarching concept for coordination and control, not just of individual DER devices, but at the level of large-scale DER/RES aggregation. 
- Show how this can be realized by common industrial, cost-effective and standardized, state-of-the-art ICT platform solutions.

- Demonstrate its practical validity via three field demonstrations covering the full range of different operating conditions including:

- normal operating conditions of DER/RES aggregations, showing their potential to reduce grid power imbalances, optimize local power and energy management, minimize cost etc. (see Sec. 3.1).

- critical operating conditions of DER/RES aggregations, showing stability when grid-integrated (see Sec. 3.2).

- emergency operating conditions, showing self-healing capabilities of DER/RES aggregations (see Sec. 3.3).

The project will summarize its IIDC reference solution by wrapping up the lessons learned from the demonstrators, and providing resulting practical how-to-build industrial guidelines. Figure 1 depicts the positioning of the INTEGRAL project.

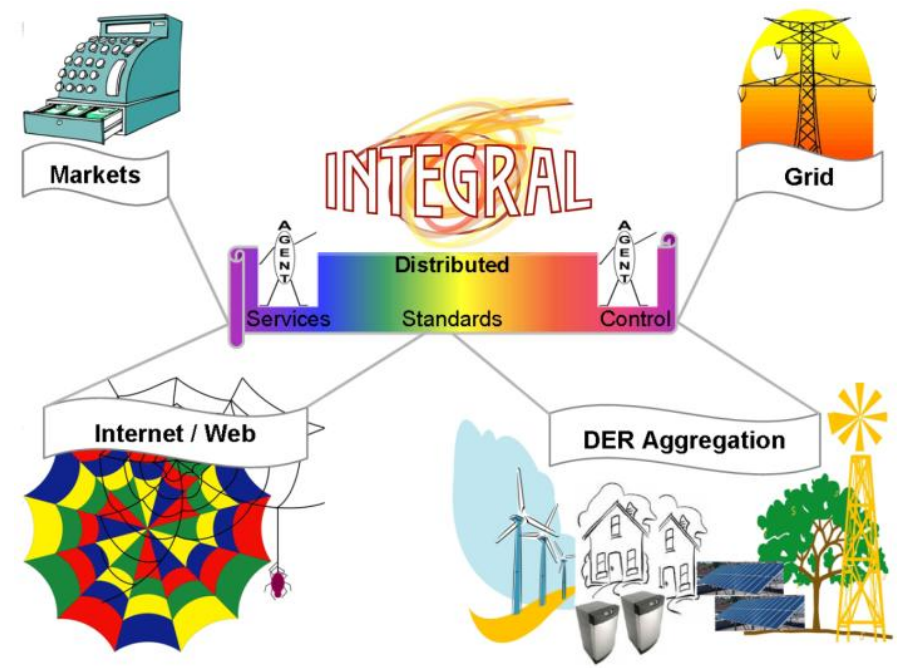

Figure 1: Positioning of the INTEGRAL project

\section{Integrated ICT platform for Distributed Control: Design Issues}

A wide range of projects in [1-4] and outside [5-7] Europe investigate the many issues that come with Smart Grids. Smart grids are grids equipped with distributed ICT to achieve a common optimization goal via an ICT enabled application. Smart or intelligent in this sense means operating grids using extended context information from the level of individual devices to the highest HVDC connection level. Intelligent also means application of results of ICT research like knowledge and agent systems and advanced computational techniques like neural networks. Compared to other approaches for making power grid applications interoperable, INTEGRAL's IIDC solution focuses on a granular, dispersed lower grid level for facilitating intelligence. 
The focus of the INTEGRAL project is to provide a set of three solutions for Active Distribution Grids enabling DER/RES integration:

- Aggregation
- Dynamic real-time context
- Cells, micro-grids
- Virtual power plants

- Integration of these DER aggregations into

- Local distribution grid operations

○ Higher-level grid operations

- Availability of

- Power trading
- Practical aggregation mechanisms
- Low-cost and industry-quality standard solutions

These Active Distribution Networks should support the following operational stages:

- Normal operations

○ Trading optimization (supplier)

- Grid optimization (DSO)

- Critical operations

$$
\text { - Support stability higher level grid }
$$

- Emergency/black start behaviour
○ Self-healing reaction to local faults
- Micro-grid mode

In addition, general requirements regarding ICT Systems in Smart Grids apply:

- Scalability
- Large numbers of DER/RES components
- Spread over large area
- Centralized control reaches complexity limits

- Openness
○ DER/RES units connect and disconnect without any central control involved
- All (future) DER/RES types must be able to connect

- Multi-actor interaction

$\begin{array}{ll}\circ & \text { Balancing of stakes: locally and globally } \\ \circ & \text { Coordination exceeding ownership boundaries } \\ \circ & \text { Decide locally on local issues (autonomy) }\end{array}$

- $\quad$ Alignment with Liberalized Energy Markets

○ Support different types of regional markets

- New market designs, especially facilitating mechanisms for better valorisations of DER/RES should be configurable

- Clustering of interests should be possible at several levels

The INTEGRAL technology consists of software and a hardware shell with applications coupled to the grid to ICT networks and business systems in a tighter or looser way depending upon the type of application. In using ICT in Power Systems, 
one can use tightly bound layers parallel to the physical infrastructure or loosely coupled layers built using existing mainstream ICT technology and infrastructures. Tightly coupled layers will be used for direct, fast control (e.g. deterministic switching in real-time); loose coupling for coordination purposes (supply-demand matching). The art of architecture design of ICT in Smart Power Grids lies in attributing a limited number of low-level functions directly coupled to the physical infrastructure and designing functionally rich applications using the loosely coupled networks. To take the INTEGRAL project as an example, for the normal operation case the link to business optimization objectives is strong as is the aggregation diversity in the cluster; the link to the physical grid is loose. On the other hand, for emergency operations the ICT grid connections should be tight and secure and the business objectives are loosely coupled as is the aggregation diversity. The critical case is inbetween these two.

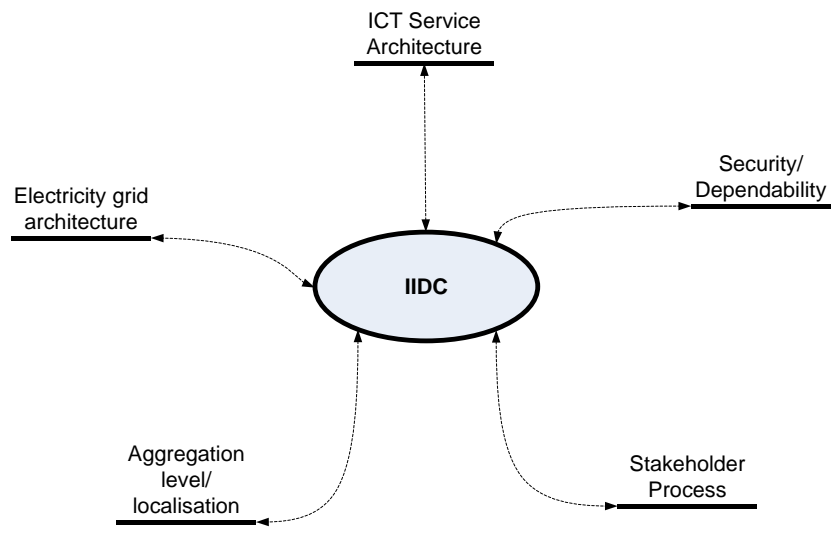

Figure 2: Five views context of ICT enabled electricity grid applications

A first context scheme for the INTEGRAL IIDC framework is shown in Figure 2. Smart Grid applications combine different architectural views in one IIDC platform:

a) The physical electricity grid architecture. This architecture includes additional ICT tightly coupled to the grid to exert direct grid control functions like real-time balancing, frequency and voltage control.

b) The ICT service architecture is loosely coupled to the grid functions. The grid is one of the context items for the ICT applications, but the operational aspects of the grid do not have to be the main issue in designing these functions.

c) The aggregation/localization level. The hierarchical division (HV/MV/LV) of the power grid is an example of aggregation on the physical level; on the application level, software enabled transparency enables variable aggregation schemes. This is one of the strong points of applying ICT in the electricity field [8]. E.g. operating a nationwide spread cluster of $\mu$-CHP might enable profitable operation on the electricity market. The nano-level refers to the individual households, the micro-level to a LV-grid comparable Microgrid, the meso-level to the MV-comparable level of a region and the macro-level to the nationwide HV-grid level issues.

The following additional aggregation levels are possible: 
- Logical aggregation: connected to certain device logics (type of apparatus)

- Functional aggregation: connected to a certain service or function in the grid. Energy and capacity based applications may lead to this type of aggregation.

- Business aggregation: linked to business objectives in a business model [2].

- Stakeholder aggregation.

A more grid oriented classification uses levels 0,1 and 2 [1, D2.1], where 0 corresponds to the above-mentioned macro level, 1 to the MV-level and 2 to the micro-grid level.

d) The stakeholder process. The process involving the stakeholder is another context boundary. Stakeholders include commercial parties optimizing portfolios for cost, installation owners, distribution system operators, home owners etc. Aggregation levels may exist. For instance, an individual housing corporation may aggregate a heat pump cluster of residents and coordinate operation. A market party may aggregate a number of Housing Corporations.

e) Security and dependability. These issues in the power system play an important role from the electrical as well as the ICT point of view.

\section{Demo Site Descriptions}

\subsection{Demo Site A - Netherlands (normal operation)}

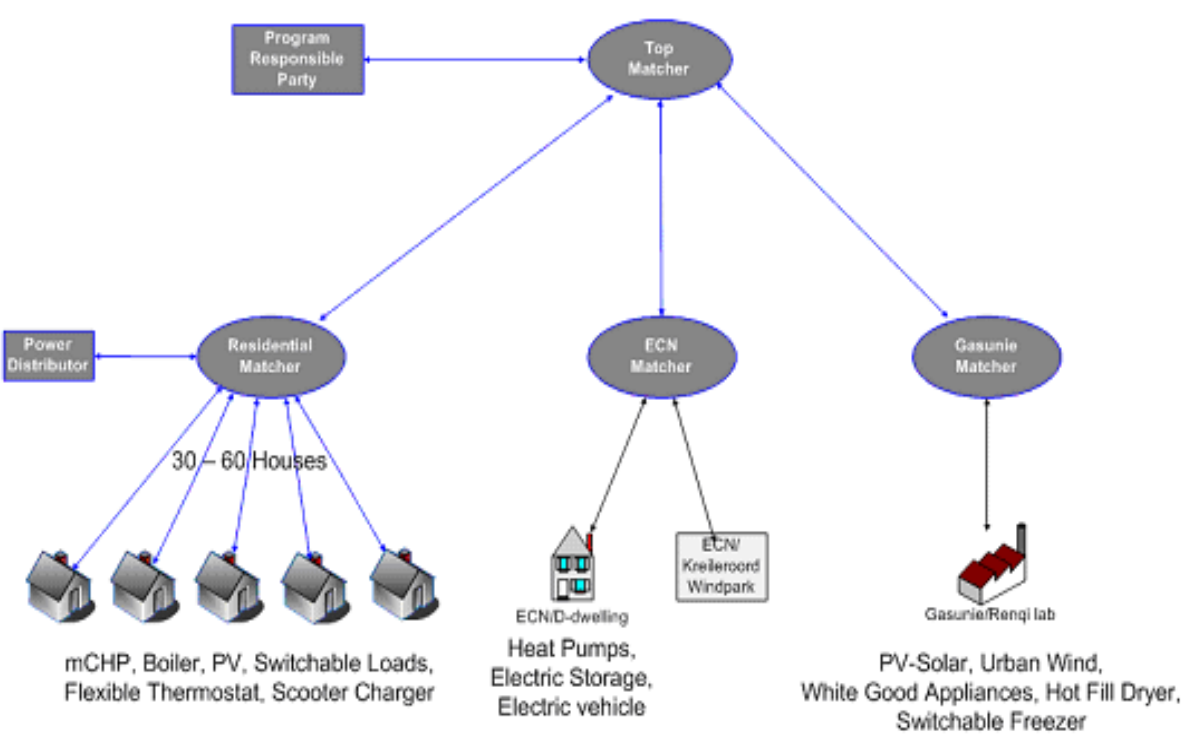

Figure 3: The PowerMatching city: Experiment A field-test configuration (normal operations) 
The normal operation 'PowerMatching City' [9,10] (Hoogkerk, NL, see Figure 3) field-test A has three hierarchic levels. At the first level, a cluster of residential houses is configured, in which generation and load flexibility is delivered by the heating systems in the houses. Through a storage tank, these are able to buffer hot water and thus heat. A flexible coupling to electricity production/consumption in the case of the heat pump and the micro-CHP is possible in this way. The heat pump can produce hot water by electricity or by an additional gas-fired peak burner in a common storage tank. The hot water can be used for heating and to produce tap water. The microCHP, when used for heating, produces electricity and heat, which can either be directly used in the house or can be buffered in the tank. In this sub-cluster setting, $\mathrm{PV}$ is a must-run generator and must-run loads are lighting loads. A power distributor agent monitors constraints on the LV distribution network. Finally the lowest level for coordination is the home context level, operation of devices behind-the-meter.

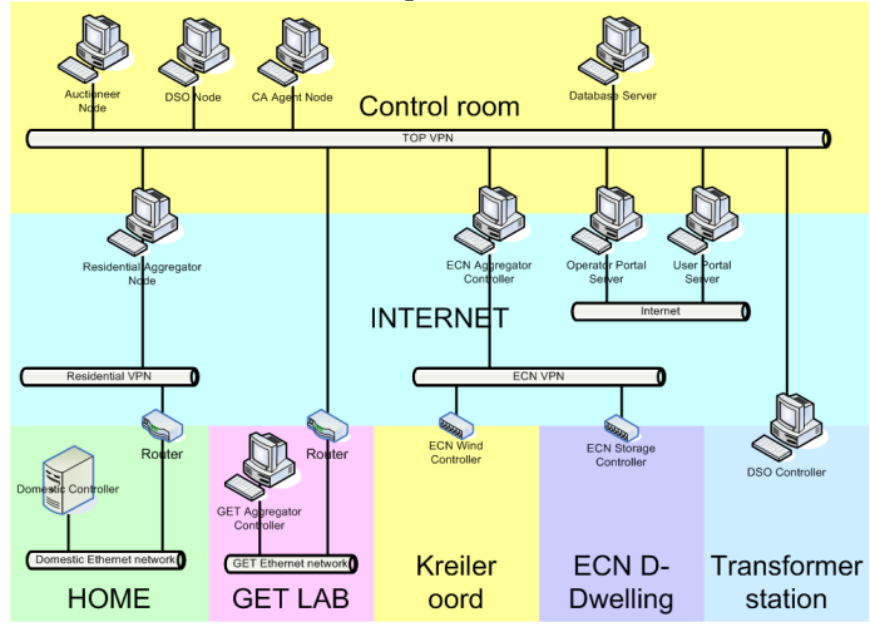

Figure 4: Experiment A communication network

The communication network infrastructure of experiment A is depicted in Figure 4. Standard TCP/IP technology is used with the public Internet protected by VPN. At the household level, a dedicated ADSL connection provides for broadband connectivity. This connectivity is also used for operation of the database, that is extensively used as a medium for information exchange in the experiment A system. Through the choice of a loosely coupled database as the cornerstone of the information system, it is possible to refine the control algorithms using already measured data from the experimental configurations. A two-level data-collection scheme is used with local fast data acquisition and processing and storage of averaged and derived data. The database used is standard SQL Server technology from Microsoft, which also has functionality for distributed processing. The data exchanged and stored include:

- $\quad$ Network set-up definitions

- $\quad$ Site set-up definitions

- Values of measurement and control signals

- PowerMatcher messages

- Configuration

- $\quad$ Logging (Log4Net) 
User interaction, interfacing hardware and inter-process communication in field-test $\mathrm{A}$ is realized in the $\mathrm{C} \#$ environment utilizing the Microsoft .NET framework supported by an SQL based relational database system. It is foreseen that implementations with Web Services using SOAP and XML will be used for interfacing the components.

\subsection{Demo Site B - Spain (critical conditions)}

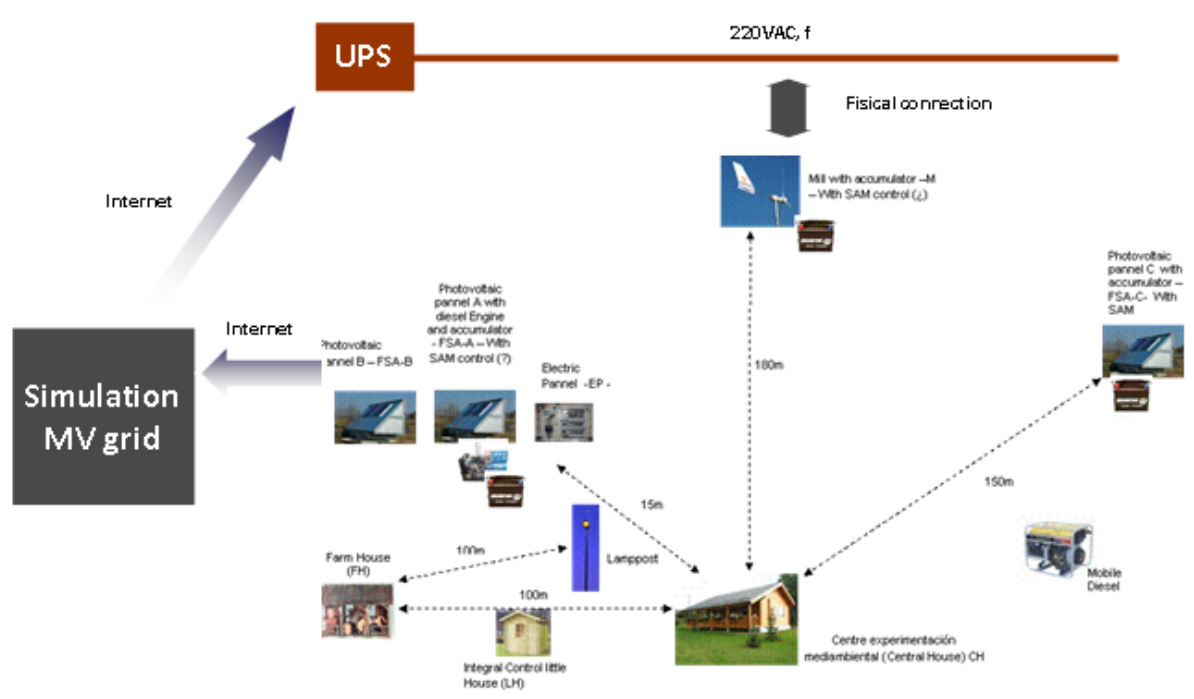

Figure 5: Experiment $B$ - the Mas Rog configuration (critical conditions)

The experiment B critical operation field-test configuration is depicted in Figure 5. There is a Microgrid, consisting of a number of RES generators (PV, Wind turbine), a backup power diesel unit and a number of shiftable loads related to domestic consumption like heating/cooling and lighting. There is a $220 \mathrm{~V}$ local grid, connected to two types of inverters including SMA-inverters, that are able to exert control actively in response to voltage and frequency deviations. As a secondary response a UPS-storage unit is available; this also contributes to produce variations on physical characteristics of the general grid in order to emulate external events. Within the Microgrid, not connected to the main grid, a separate place is reserved for additional equipment, which emulates external events (due to legislation in Spain, the Microgrid is not allowed to be physically connected to the main grid).

In field-test B supply-demand matching coordination is performed to react to critical situations in the grid. The supply-demand matching is done using software agents operating in an agent development and execution environment. In this environment, JADE, device behaviour and energy usage optimization are also at stake. Embedded controllers in the field (ZigBee nodes) are linked to a central PC in which the agents are operating as separate software processes. Therefore, having a JADE $<>$ WCF/.NET interface would enable flexible interchange of agent logics and data. A DataBase Agent (DBA) provides the functionality on the use of the general 
database, a User Agent (UA) provides the communication with the User, and finally the Local Controller (LC) is the agent assigned to the specific element in the field with its particular logic for the device.

The software interconnectivity in field-test B is realized by ZigBee technology for real field communication and basic reactive control in the hardware part, and by using primitives from the JADE environment in the software part for complex control. Each device has physically associated a unique ZigBee node which is communicated to the central system. Into the central system, each device in the real field has an associated agent which is part of the multi agent system (MAS). Thus, the interconnectivity in field-test $\mathrm{B}$ is realized using primitives from the JADE environment.

The ZigBee to agent connection (the MAS system) is established using the serial RS-485 protocol via a ZigBee mesh network by means of the central ZigBee node connected to the multi agent system. Communication is via the Microgrid Central Controller (MGCC). The Micro Grid Central Controller (MGCC) provides the coordination of the system. This Microgrid Central controller has communication with the Internet by means of standard TCP/IP protocol, cf. Figure 6 .

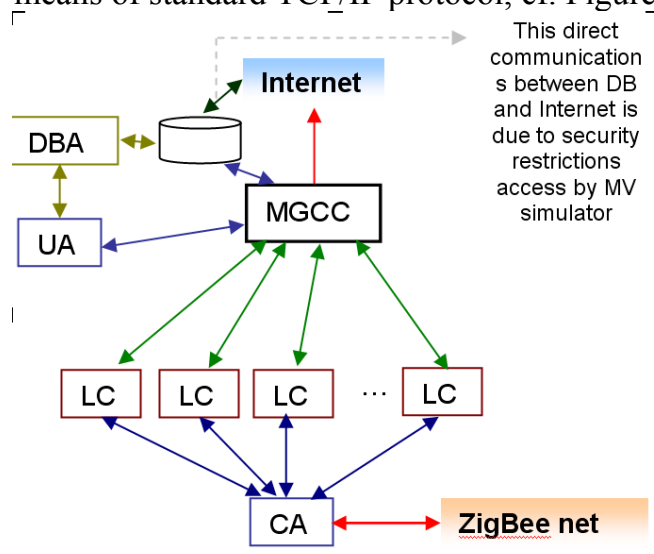

Figure 6: Communication network of experiment B

\subsection{Demo Site C - France (emergency case)}

The emergency case field-test $\mathrm{C}$ configuration is depicted in Figure 7. Use cases are the following:

Fast fault detection and isolation (location robustness, fault type). On the demonstration network, different fault locations and short circuits will be tested (three phases, two phases, single phase with or without grounding). The DSO will use the high level functionality associated with the ADA devices (Fault Passage Indicator, remote control switches...) in order to quickly detect and isolate the faulty section.

Fast service restoration processes (communication performance). Short circuit and self healing functions will be tested. The performance of the communication, bandwidth and latency will be studied between the RTUs and the agents.

Fast fault detection and isolation (grounding of the substation). Short circuits (of a different nature) and self healing functions will be tested as function of grounding 
type. The DSO will then be able to quickly detect and isolate the faulty section even in various grounding conditions.

Fast fault detection and isolation (load and generation conditions). On the demonstration network, different short circuits will be tested with various loading and sourcing conditions.

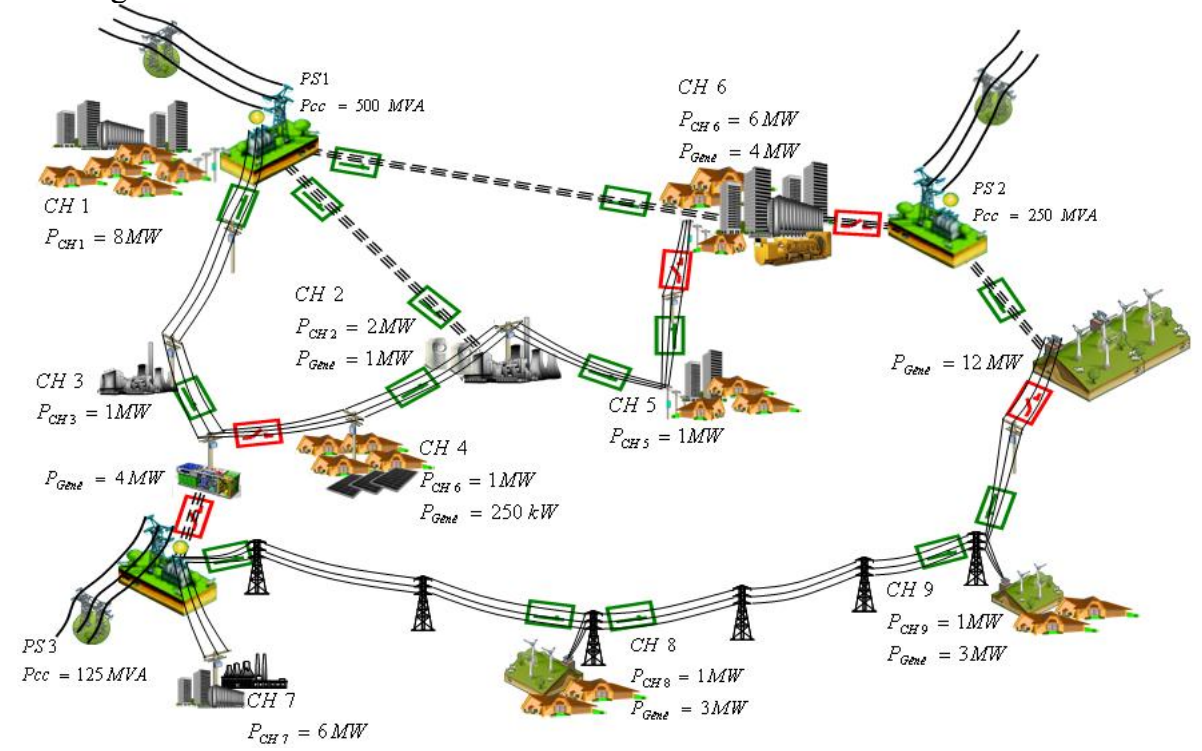

Figure 7: Experiment $C$ configuration (emergency operations)

The field-test $\mathrm{C}$ communication configuration is shown in Figure 8. Equipment and tools used are based on standard open protocols with interfaces to proprietary protocols. OLE for Process Control $\left(\mathrm{OPC}^{\mathrm{TM}}\right)$ via Ethernet is used as the standard protocol for communication between the SCADA OPC server and the RTUs (OPC clients). Equipment and tools used are:

- The electrical measurement devices: current transducer (4..20mA), voltage transducer (4..20mA) linked with the automated components and the RTUs will be installed to have the real situation of the experimental network.

- The communication requirements of the different elements will be analyzed.

- The Intelligent Ethernet Switches from BTH will be installed and validated.

- Communicating RTUs based on the PLC/PC104 standard will be analyzed and chosen in term of required data acquisition and computerization abilities.

- $\quad$ OPC servers need to be installed in the ICT platform of G2ELab laboratory.

- A link between OPC servers and OPC MATLAB toolbox.

- A link between the MATLAB toolbox and advanced functions for self healing is established.

Closed loop control between MATLAB advanced functions and SCADA system are being designed to operate the remotely controlled switches. The agent logics is coupled through the OPC server. 


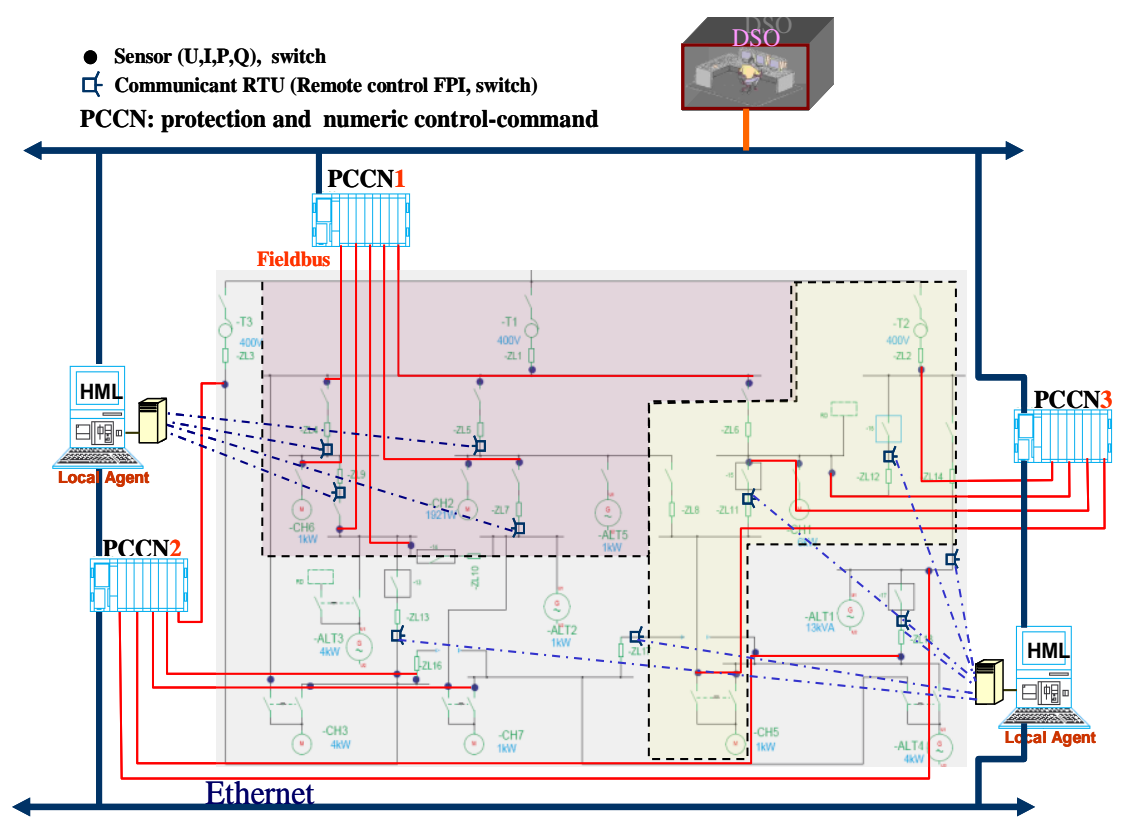

Figure 8: Communication network of Experiment C

\section{Further information}

Further information on the EU project INTEGRAL, including the IIDC common architecture and results of the field experiments, will be made available on the project website at http://www.integral-eu.com.

\section{References}

1. CRISP, 2003 Deliverables can be found at http://crisp.ecn.nl

2. e3value, 2008 See www.e3value.com (see e.g. the BUSMOD project)

3. Fenix, $2008 \mathrm{http} / / / \mathrm{www}$. fenix-project.org/

4. Flexibel, 2008 Detailed info at http://www.flexible-electricity-networks.nl/

5. GridWise, $2008 \mathrm{http}$ //Www.gridwise.org/

6. Intelligrid, 2003 See www.iec.ch for the standard texts

7. NETL, 2008 See http://www.netl.doe.gov/moderngrid/

8. FPS, 2005 R. Kamphuis et al. (ECN): Software Agents for Matching of Power Supply and Demand: A Field-test with a Real-time Automated Imbalance Reduction System, in Future Power Systems 2005, Nov. 2005, Amsterdam, NL.

9. Gasunie, $2007 \mathrm{~A}$ field test using agents for coordination of residential $\mu$-CHP.

10. C.J. Warmer et al.: Intelligent Systems Applications to Power Systems. ISAP 2007 Proceedings , 5-8 Nov. 2007, pp. 1-4. 